\title{
Macro and Micronutrients of 44 Medicinal Plant Species Used Traditionally in Nuevo Leon, Mexico
}

Ratikanta Maiti ${ }^{1 *}$, Humberto Gonzalez Rodriguez ${ }^{1}$, Hewan Demissie Degu ${ }^{2}$, Ch. Aruna Kumari ${ }^{3}$ and N. C. Sarkar ${ }^{4}$

${ }^{1,2}$ Universidad Autonoma de Nuevo Leon, Facultad de Ciencias Forestales, Carr. Nac. No. 85 Km. 45, Linares, Nuevo Leon (67 700), Mexico

${ }^{2}$ School of Plant and Horticulture Science, Plant Biotechnology, Hawassa, SNNPR (1417), Ethiopia, ${ }^{3}$ PJTSAU, Agricultural College, Polasa, Jagtial, Karimnagar (505 529), India

${ }^{4}$ Dept. of Agronomy, Institute of Agriculture, Visva-Bharati, Sriniketan, Dist- Birbhum, West Bengal (731 236), India

\section{Article History}

Manuscript No. AR1638a

Received in $10^{\text {th }}$ July, 2016

Received in revised form $25^{\text {th }}$ September, 2016

Accepted in final form $29^{\text {th }}$ September, 2016

\section{Correspondence to}

*E-mail: humberto.gonzalezrd@uanl.edu.mx

\section{Keywords}

Medicinal plants, variability, macro and micronutrients, nutraceuticals

\begin{abstract}
An analysis of macro and micronutrients among 44 species of medicinal plants utilized traditionally to control diabetes and other diseases in Nuevo Leon, Mexico carried out at the experimental station of Facultad de Ciencias Forestales, Universidad Autonoma de Nuevo Leon, located in the municipality of Linares showed a large variability in the contents of macro and micronutrients and protein. $\mathrm{K}$ exhibited a wide range of variability among the selected medicinal plants 5.47-163.35 ( $\left.\mathrm{mg} \mathrm{g}^{-1} \mathrm{dw}\right), \mathrm{Mg}$ from 0.1-8.64 (mg g $\mathrm{mw}^{-1}$ ), P varied from $0.18-5.79\left(\mathrm{mg} \mathrm{g}^{-1} \mathrm{dw}\right)$, C from $25.54-51.66 \%$, $\mathrm{N}$ from $1.36-6.25 \%, \mathrm{C} / \mathrm{N}$ from $5.34-31.05 \%$ and with respect to micronutrients $\mathrm{Cu}$ ranged from 4.17-33.88 $\left(\mu \mathrm{g} \mathrm{g}^{-1} \mathrm{dw}\right)$, Fe from 98.28-3977.55 $\left(\mu \mathrm{g} \mathrm{g}^{-1} \mathrm{dw}\right), \mathrm{Zn}$ from 9.49-266.31 ( $\left.\mu \mathrm{g} \mathrm{g} \mathrm{g}^{-1} \mathrm{dw}\right)$. It is assessed that some species contain high amount of nutrients (macro and micronutrients thereby confirming their efficacy to combat various diseases and provide an opportunity to scientists working on medicinal plants to select the species with high nutrient contents.
\end{abstract}

\section{Introduction}

Since, remote past traditional medicinal plants have been used to alleviate various diseases in the different countries. Mexico represents one of the countries exhibiting the highest diversity of plants in the World (Mittermeier, 1988; Mittermeier and Mittermeier, 1992; Villasenor, 2003). In Mexico, traditional medicinal plants are commonly used in rural and urban areas to cure various diseases (Mittermeier, 1988; Ankli, 2000; Villasenor, 2003; Vega-Avila et al., 2009). Although various studies have been undertaken on ethno-botany of medicinal plants (Bell and Castetter, 1937), only few cases were reported in the north-eastern area of Mexico (Hernandez-Sandoval et al., 1991; Estrada et al., 2007. What is more, very little information is available to determine the efficacy of each species on the basis of chemical analysis.

Various studies have been undertaken on the use of micro and macronutrients in alleviating human and animal diseases, such as in alleviating eye problem (Kowluru et al., 2008); diabetes (Patton et al., 2007, Farvid et al., 2011). Different micronutrients, although required in minor quantities are essential for good health of mankind and animals. The deficiency of these elements causes abnormalities leading to infection of diseases. Several medicinal plants possess micronutrients and are useful in diabetes. Few medicinal plants are reported to be effective to control blood sugar such as Mulberry leaf, soybean, black bean, Persian shallot, Zizyphus lotus, etc. Some Mexican plants contain $\beta$-glycosides and other compounds that are effective in lowering blood sugar and body weight in diabetic patients. It has been reported that carbon, nitrogen and $\mathrm{C} / \mathrm{N}$ ratios are related to the production of secondary metabolites, antioxidants and flavonoids which are useful for the health care of human beings (Devangyam et al., 2004, Safrini et al., 2002). Antioxidants reduce inflammatory properties, promote cardiovascular health (Monde and Youdin, 2004), inhibits cancerous tumour (Shoskes et al., 1999), and reduce ageing process in the brain and nervous system (Shaheen et al., 2001) and delay or inhibit oxidation (Halliwal and Gutteridge, 1989). Antioxidant compounds are present in fruits, vegetables viz., Occimum sanctum, Terminalia belerica, Zingiber officinales and several Chinese and Indian spices such as Allium sativa. Majority of the antioxidant activities are contributed by flavones, isoflavones, flavonoids, anthocyanins, coumarinsligans, catechin and isocatechin found in plants 
(Schramm et al., 2003). High peroxidation of secondary metabolites was detected in plants with high $\mathrm{C} / \mathrm{N}$ ratio and low nitrogen fertilization especially when exposed to elevated $\mathrm{CO}_{2}$ levels. Under low nitrogen conditions, the growth and photosynthesis in plant increases the $\mathrm{C} / \mathrm{N}$ ratio which intern increase the production of secondary metabolites (Shaheen et al., 2001; Lindroth et al., 2002.

In medicinal plants, litter degradation was found to be affected by the UV radiation. Elevated influx of ultraviolet-B radiation (UV-B) as a consequence of depletion of stratospheric ozone $\left(\mathrm{O}_{3}\right)$ layer may affect litter decomposition directly/modifying the plant tissue quality. UV-B modified the decomposition rate of leaf litter of test medicinal plant species, altered strongly the tissue chemistry particularly leaf phenolic, $\mathrm{N}$ and $\mathrm{P}$ concentrations and affected the decay rate which was species specific (Agrawal and Kumari, 2013). Very recently strong recommendation for consumption of nutraceutical from plant has become popular to improve health and to prevent/ treat various diseases. Some popular phyto-nutraceuticals include glucosamine from ginseng Omega 3 fatty acids from linseed. Many of nutraceutical have high potentials for multiple therapeutic, but research is lacking (Pandey et al., 2011).

Many researchers revealed the usage of medicinal species traditionally in controlling particular diseases, very little research inputs are directed in determining the efficacy of medicinal species with respect to their nutritional contents viz., macronutrient or micronutrient contents. It is well documented that the presence of macronutrients or micronutrient in a medicinal plant species is very important in alleviating its utility to combat diseases. The chemical analysis of macronutrients or micronutrients in these medicinal plant species is rarely reported. Maiti et al. (2015) has reported macro and micronutrients of 18 medicinal plants used traditionally for Diabetes in Mexico. Hence, the present study was undertaken to determine micronutrients $(\mathrm{Cu}, \mathrm{Fe}$ and $\mathrm{Zn})$ and macronutrients ( $\mathrm{K}, \mathrm{Mg}$ and $\mathrm{P}$ ), $\mathrm{C}, \mathrm{N}$ and $\mathrm{C} / \mathrm{N}$ ratio of 44 medicinal plants used in Nuevo Leon in Northeast of Mexico and select plants with high nutritional value (macro and micronutrient contents) for high efficacy.

\section{Materials and Methods}

This study was carried out at the experimental station of Facultad de Ciencias Forestales, Universidad Autonoma de Nuevo Leon, located in the municipality of Linares $(2447 \mathrm{~N} .9932 \mathrm{~W})$, at elevation of $350 \mathrm{~m}$. The climate is subtropical or semiarid with warm summer, monthly mean air temperature vary from $14.7{ }^{\circ} \mathrm{C}$ in January to $23{ }^{\circ} \mathrm{C}$ in August, although during summer the temperature goes up to $45^{\circ} \mathrm{C}$. Average annual precipitation is around $805 \mathrm{~mm}$ with a bimodal distribution. The dominant type of vegetation is the
Tamaulipan Thorn scrub or subtropical Thorn scrub wood land. The dominant soils deep, dark grey, lime-grey, vertisol with montmorrillonite, which shrinks and swell remarkably in response to change in moisture contents.

The medicinal plants were collected from botanical gardens of Forest Science Faculty, UANL. Mexico 44 medicinal plants by the indigenous people of Mexico were used to describe the efficacy of these medicinal plants. The name of the plants and their respective usage is described in Table 1 . The study was directed in two phases, $1^{\text {st }}$ phase was Analysis of three micro $(\mathrm{Cu}, \mathrm{Fe}, \mathrm{Zn})$ and three macronutrients $(\mathrm{K}, \mathrm{Mg}, \mathrm{P})$ followed by the $2^{\text {nd }}$ phase Analysis of C, N, C/N of 26 medicinal plants used in diabetes. Nitrogen value is multiplied by a factor of 6.25 to obtain the $\%$ protein.

\subsection{Chemical analysis}

Medicinal plant samples were collected and placed to dry on newspaper for a week. The leaves were separated from the rest of the plant and were passed twice through a mesh of $1 \times 1$ $\mathrm{mm}$ in diameter using a mill Thomas Wiley and subsequently dried for more than three days at $65^{\circ} \mathrm{C}$ in an oven (Precision model 16EG) to remove moisture from the sample and later these were placed in a desiccators. A $2.0 \mathrm{mg}$ of the sample was weighed in an AD6000 Perkin balance Elmer in a vial of tin, bent perfectly. This was placed in Chonsanalyzer Perkin Elmer Model 2400 for determining carbon, hydrogen and nitrogen. For estimating the mineral contents, the samples were incinerated in a muffle oven at $55^{\circ} \mathrm{C}$ for 5 hours. A shed sample is digested in a solution containing $\mathrm{HCL}$ and $\mathrm{HNO}_{3}$, using the wet digestion technique (Cherney, 2000). Carbon and nitrogen foliar contents ( $\%$ dry mass basis) were carried out in $0.02 \mathrm{~g}$ of milled dried leaf tissue by using a CHN analyser.

\section{Results and Discussion}

We determined macronutrients $(\mathrm{K}, \mathrm{Mg}, \mathrm{P}, \mathrm{C}, \mathrm{C} / \mathrm{N})$, protein content, and micronutrients $(\mathrm{Cu}, \mathrm{Fe}, \mathrm{Zn})$ which from the 44 leaves of medicinal plants (Table 2). The information helps to select plants with high quantity of each nutrient. This will intern confirm the efficacy of the medicinal activity of the plant. An analysis of macro and micronutrients showed a large variability. The present study showed a large variation in the contents of nutrients and protein $\%$, thereby offering opportunity by scientists working on medicinal. 26 medicinal plants were grouped based on the maximum content of macro and micro nutrients.

\subsection{Macronutrients}

With respect to macronutrients, $\mathrm{K}$ varied from 5.47-163.35 ( $\left.\mathrm{mg} \mathrm{g}^{-1} \mathrm{dw}\right), \mathrm{Mg}$ from $0.17-8.64\left(\mathrm{mg} \mathrm{g}^{-1} \mathrm{dw}\right), \mathrm{P}$ varied from $0.18-5.79\left(\mathrm{mg} \mathrm{g}^{-1} \mathrm{dw}\right), \mathrm{C}$ from $25.54-51.66 \%$, N from $1.36-6.25 \%, \mathrm{C} / \mathrm{N}$ from $5.34-31.05$ and with respect to 


\begin{tabular}{|c|c|c|c|c|}
\hline Particular disease & Common name & Scientific name & Family & Type \\
\hline Diabetes & Maguey Todaro & Agave macroculmis Todaro & Agavaceae & Rosetofilus \\
\hline Diabetes & Madrono & Arbutus xalapensis & Ericaceae & Bush \\
\hline Diabetes & Pata de vaca & Bauhinia forficata & Fabaceae & Tree \\
\hline Diabetes & Tepozan & Buddleja cordata & Buddlejaceae & Tree \\
\hline Diabetes & Nogal & Carya illinoiensis & Juglandaceae & Tree \\
\hline Diabetes & Palo blanco & Celtis laevigata & Ulmaceae & Tree \\
\hline Diabetes & Canela & Cinnamomum verum & Lauraceae & Tree \\
\hline Diabetes & Salvia & Croton suaveolens & Euphorbiaceae & Bush \\
\hline Diabetes & Níspero & Eryobotria japonica & Rosaceae & Bush \\
\hline Diabetes & $\begin{array}{l}\text { Betonica o poleo de } \\
\text { hoja ancha }\end{array}$ & Hedeoma palmeri & Lamiaceae & Bush \\
\hline Diabetes & Manrrubio & Marrubium vulgare & Lamiaceae & Herb \\
\hline Diabetes & Neem & Melia azadirachta & Meliaceae & Tree \\
\hline Diabetes & Moringa & Moringa oleifer & Moriginaceae & Tree \\
\hline Diabetes & Nopal de t. año & Opuntia ficus-indica & Cactaceae & Bush \\
\hline Diabetes & Injerto & Phoradendron villosum & Viscaceae & Bush \\
\hline Diabetes & Lantrisco & Rhus virens & Anacardiaceae & Bush \\
\hline Diabetes & Sauce & Salix lasiolepis & Salicaceae & Tree \\
\hline Diabetes & Tronadora & Tecoma stans & Bignoniaceae & Bush \\
\hline Diabetes & Maguey Todaro & Agave macroculmis Todaro & Agavaceae & Rosetofilus \\
\hline Ulcer, tumour, respiratoryproblems & Una de gato & Acacia wrightii & Mimosaceae & Tree \\
\hline Cancer & Hierba del cancer & Acalypha monostachya & Euphorbiaceae & Herb \\
\hline Bronquitis, , infertility,inlamatiion & Hierba de San Nicolás & Chrysactinia mexicana & Asteraceae & Bush \\
\hline Circulation, digestion & Chaya & Cnidoscolus aconitifolius & Euphporbiaceae & Tree \\
\hline Fever & Parraleña & Dyssodia setifolia & Asteraceae & Herb \\
\hline Kidney, cancer & Cola de caballo & Equisetum hyemale & Equisetaceae & Stemerect \\
\hline Stomache & Colesia & Eruca sativa & Brasscaceae & Herb \\
\hline Cholesterol & Hierba del sapo & Eryngium heterophyllum & Apiaceae & Herb \\
\hline Tonic, stimulant & Clavo de olor & Eugenia caryophyllata & Myrtaceae & Tree \\
\hline Expectorant & Gordolobo & Gnahalium canescens & Asteraceae & Herb \\
\hline Stomache & Ocotillo & Gochnatia hypoleuca & Asteraceae & Bush \\
\hline Stomache & Hierba del pajarito & Lepidium virginicumm & Brasscaceae & Herb \\
\hline Stomache & Laurel & Litsea glauscesens & Lauraceae & Bush \\
\hline Stomache & Yerbabuena & Mentha piperita & Lamiaceae & Herb \\
\hline Kidney & Charrasquilla & Mimosa malacophylla & Leguminosae & sub Bush \\
\hline Headache, gum pain, joint pain & Gigante & Nicotiana glauca & Solanaceae & Bush \\
\hline Earache, taquicardia & Albahaca & Ocimum basilicum & Lamiaceae & herb \\
\hline Cholesterol & Olivo & Olea europea & Oleaceas & Tree \\
\hline Circulation, bloodpressure & Alpistle & Phalaris canariensis & Poaceae & Bush \\
\hline Cough, expectorante & Oregano & Poliomintha longiflora & Lamiaceae & Bush \\
\hline Circulation, hairfall & Romero & Rosamrinus officinalis & Lamiaceae & Bush \\
\hline
\end{tabular}




\begin{tabular}{lllll}
\hline Particular disease & Common name & Scientific name & Family & Type \\
\hline Earache, bloodpressure & Ruda & Ruta graveolens & Rutaceae & Herb \\
$\begin{array}{l}\text { Cancer, coagulation, antitumourr, } \\
\text { elimination of fat }\end{array}$ & Chia & Salvia hispanica & Lameaceae & Herb \\
Somache, antifunga & Tatalencho & Tagetes lucida & Asteraceae & sub Bush \\
Burning & Paistle & Tillandsia usenoides L. & Bromeliaceae & Caulescent \\
Bronchitisbloodpurification & Ortiguilla & Tragia ramosa & Euphporbiaceae & Herb \\
\hline
\end{tabular}

micronutrients $\mathrm{Cu}$ ranged from 4.17-33.88 ( $\left.\mu \mathrm{g} \mathrm{g}^{-1} \mathrm{dw}\right)$, Fe from 98.28-3977.55) ( $\left.\mu \mathrm{g} \mathrm{g}^{-1} \mathrm{dw}\right), Z n$ from 9.49-266.3 ( $\left.\mu \mathrm{g} \mathrm{g}^{-1} \mathrm{dw}\right)$.

Potassium is an electrolyte, a substance that conducts electricity in the body. $\mathrm{K}$ is crucial to heart function and plays a key role in skeletal and smooth muscle contraction, making it important for normal digestive and muscular function. K contents in some of the plant leaves are Phalaris canariensis (163.3), Eruca sativa (144.2), Opuntiaficus-indica (101.5), Phoradendron villosum (100.6), Moringa olelfer (96.0), Marrubium vulgare (91.3), Melia azadirachta (100), Agave macroculmis Todaro (78.5), Hedeoma palmeri (76.50), Croton suaveolens (75.6), Ocimum basilicum (72.1).

Magnesium is involved in hundreds of enzyme reactions in the body as it performs an array of biological functions as activation of muscles and nerves, digestion of proteins, carbohydrates, fats, building block for RNA and DNA synthesis. Mimosa malacophylla (8.6), Acalypha monostachya (8.1), Opuntia ficus-indica (6.4), Salvia hispanica (3.5), Melia azadirachta (3.41), Phoradendron villosum (2.3), Eryobotriav japonica (2.2) contain higher amounts of $\mathrm{Mg}$.

The role of phosphorus in energy transfer is well known, it is also help for the growth and repair of the cells. Phosphorus gives structure and strength to the body. The important $\mathrm{P}$ contain species are Celtis laevigata (4.03), Carya illinoiensis (2.89), Croton suaveolens (2.40), Litsea glauscesens (2.40), Eryngium heterophyllum (2.27), Ruca sativa (2.22), Lepidium virginicumm (2.27), Cnidoscolus aconitifolius (2.05), Mentha piperita (2.0).

Eugenia caryophyllata (51.66), Rhus virens (50.38), Cinnamomum verum (49.34), Arbutus xalapensis (49.1), Tecoma stans (48.79), Eryobotria japonica (47.98), Rosamrinus officinalis (47.77), Hedeoma palmeri (46.38), Moringa olelfer (45.96), Buddleja cordata (45.70), Croton suaveolens (45.17), Mimosa malacophylla (45.15), Chrysactinia Mexicana (45.04), Melia azadirachta (45.12) are the species contain higher carbon.

Nitrogen content varies mentioned as Mimosa malacophylla (8.46), Moringa oleIfer (6.25), Tagetes lucida (5.98), Tagetes lucida (5.89), Marrubium vulgare (5.58), Eruca sativa (5.48), Salvia hispanica (5.24), Mentha piperita (3.4), Phoradendron villosum (4.92), Poliomintha longiflora (4.89), Nicotiana glauca (4.79), Ocimum basilicum (4.66), Marrubium vulgare (4.56), Rosamrinus officinalis (4.54), Lepidium virginicumm $(4,40)$, Cnidoscolus aconitifolius (4.34).

It has been reported by various authors that Carbon, Nitrogen and $\mathrm{C} / \mathrm{N}$ ratio are related to the production of secondary metabolites and antioxidants, flavonoids related to health care of human beings.

The present study indicated that the species selected for high $\mathrm{C} / \mathrm{N}$ ratio viz. Agave macroculmis (31.04), Arbutus xalapensis (26.94), Eryngium heterophyllum (24.23), Rhus virens (22.92), Olea europea (22.08), Croton suaveolens (20.16), Hedeoma palmeri (17.14), Salix lasiolepis (16.26), Acalypha monostachya (15.95), Salix lasiolepis (15.17) are recommended for analysis of antioxidants and secondary metabolites. These form the future potential lines of research. The role of antioxidants has been documented in cell functions, structure, lipid leading to the non-development of chronic diseases such as cancer (Safrini et al., 2002; Devangyam et al., 2004). They provide many health benefits and reduce inflammatory properties, promote cardiovascular health (Mondel and Youdin, 2004), inhibits cancerous tumour (Shoskes et al., 1999), reduce ageing process in the brain and nervous system (Shahee et al., 2001) delays or inhibit oxidation (Halliwal and Gutteridge, 1989). Antioxidant compounds are present in fruits, vegetables such as Occimum sanctum, Terminalia belerica, Zingiber officinales and several Chinese and Indian spices such as such as Allium sativa. Majority of the antioxidant activities are attributed to flavones, isoflavones, flavonoids, anthocyanin, coumarinligans, catechin and isocatechin found in plants. The natural antioxidants have been reported over a range of compounds including flavonoids, phenolic, nitrogen compounds and carotenoids (Schramm et al., 2003). Many plant species possess antioxidant properties. Ibrahim et al. (2011) reported that high peroxidation of secondary metabolites was elicited in plants by $\mathrm{C} / \mathrm{N}$ ratio and low nitrogen fertilization especially when exposed to elevated $\mathrm{CO}_{2}$ levels. Under low nitrogen, the growth and photosynthesis in plant show increase in $\mathrm{C} / \mathrm{N}$ ratio and gives increase in the production of secondary metabolites (Shahee et al., 2001). Therefore high $\mathrm{C} / \mathrm{N}$ ratio might be attributed to low nitrogen 


\begin{tabular}{|c|c|c|c|c|c|c|c|c|c|c|}
\hline \multirow[t]{3}{*}{ Plant species } & \multicolumn{5}{|c|}{ Leaf macro-nutrient content } & \multirow[t]{3}{*}{$\mathrm{C}: \mathrm{N}$} & \multirow{3}{*}{$\begin{array}{c}\% \\
\text { Proteina }\end{array}$} & \multirow{2}{*}{\multicolumn{3}{|c|}{$\frac{\text { Leaf micro-nutrient content }}{\left(\mu \mathrm{g} \mathrm{g}^{-1} \mathrm{dw}\right)}$}} \\
\hline & \multicolumn{3}{|c|}{$\left(\mathrm{mg} \mathrm{g}^{-1} \mathrm{dw}\right)$} & \multicolumn{2}{|c|}{$(\%)$} & & & & & \\
\hline & K & $\mathrm{Mg}$ & $\mathrm{P}$ & $\mathrm{C}$ & $\mathrm{N}$ & & & $\mathrm{Cu}$ & $\mathrm{Fe}$ & $\mathrm{Zn}$ \\
\hline Agave macroculmis Todaro & $\begin{array}{c}78.45 \pm \\
1.07\end{array}$ & $\begin{array}{c}1.38 \pm \\
0.29\end{array}$ & $\begin{array}{c}0.73 \pm \\
0.02\end{array}$ & $\begin{array}{c}41.32 \pm \\
0.74\end{array}$ & $\begin{array}{c}1.36 \pm \\
0.21\end{array}$ & $\begin{array}{l}31.05 \pm \\
5.11\end{array}$ & 8.49 & $\begin{array}{c}18.76 \pm \\
2.87\end{array}$ & $\begin{array}{c}210.53 \pm \\
17.37\end{array}$ & $\begin{array}{c}55.20 \pm \\
3.39\end{array}$ \\
\hline Arbutus xalapensis & $\begin{array}{c}30.65 \pm \\
13.9\end{array}$ & $\begin{array}{c}0.54 \pm \\
0.60\end{array}$ & $\begin{array}{c}1.78 \pm \\
0.40\end{array}$ & $\begin{array}{c}49.1 \pm \\
0.42\end{array}$ & $\begin{array}{c}1.86 \pm \\
0.30\end{array}$ & $\begin{array}{c}26.94 \pm \\
3.72\end{array}$ & 11.6 & $\begin{array}{c}33.40 \pm \\
20.18\end{array}$ & $\begin{array}{c}347.58 \pm \\
104.20\end{array}$ & $\begin{array}{c}25.07 \pm \\
2.65\end{array}$ \\
\hline Bauhinia forficata & $\begin{array}{c}8.29 \pm \\
1.27\end{array}$ & $\begin{array}{c}1.45 \pm \\
0.31\end{array}$ & $\begin{array}{c}1.37 \pm \\
0.11\end{array}$ & $\begin{array}{l}34.02 \pm \\
2.32\end{array}$ & $\begin{array}{c}2.33 \pm \\
0.6\end{array}$ & $\begin{array}{c}14.60 \pm \\
3.86\end{array}$ & 14.56 & $\begin{array}{c}12.86 \pm \\
0.73\end{array}$ & $\begin{array}{c}161.65 \pm \\
5.30\end{array}$ & $\begin{array}{c}10.73 \pm \\
0.28\end{array}$ \\
\hline Buddleja cordata & $\begin{array}{l}39.30 \pm \\
1.82\end{array}$ & $\begin{array}{c}0.17 \pm \\
0.09\end{array}$ & $\begin{array}{c}0.56 \pm \\
0.10\end{array}$ & $\begin{array}{c}45.70 \pm \\
0.56\end{array}$ & $\begin{array}{c}3.26 \pm \\
0.40\end{array}$ & $\begin{array}{c}14.16 \pm \\
1.44\end{array}$ & 20.38 & $\begin{array}{c}29.90 \pm \\
1.85\end{array}$ & $\begin{array}{c}148.00 \pm \\
26.25\end{array}$ & $\begin{array}{c}40.26 \pm \\
3.39\end{array}$ \\
\hline Carya illinoiensis & $\begin{array}{l}31.16 \pm \\
1.89\end{array}$ & $\begin{array}{c}0.85 \pm \\
0.34\end{array}$ & $\begin{array}{c}2.89 \pm \\
0.06\end{array}$ & $\begin{array}{c}44.27 \pm \\
1.00\end{array}$ & $\begin{array}{c}3.76 \pm \\
0.71\end{array}$ & $\begin{array}{c}12.04 \pm \\
1.81\end{array}$ & 23.5 & $\begin{array}{c}25.74 \pm \\
1.70\end{array}$ & $\begin{array}{l}166.49 \pm \\
20.45\end{array}$ & $\begin{array}{c}57.69 \pm \\
7.74\end{array}$ \\
\hline Celtis laevigata & $\begin{array}{c}16.10 \pm \\
4.59\end{array}$ & $\begin{array}{c}1.51 \pm \\
0.67\end{array}$ & $\begin{array}{c}4.03 \pm \\
0.29\end{array}$ & $\begin{array}{c}39.45 \pm \\
0.51\end{array}$ & $\begin{array}{c}3.01 \pm \\
0.18\end{array}$ & $\begin{array}{c}13.13 \pm \\
0.70\end{array}$ & 18.83 & $\begin{array}{c}33.88 \pm \\
12.60\end{array}$ & $\begin{array}{c}213.15 \pm \\
49.80\end{array}$ & $\begin{array}{c}23.53 \pm \\
1.91\end{array}$ \\
\hline Cinnamomum verum & $\begin{array}{c}16.14 \pm \\
1.35\end{array}$ & $\begin{array}{c}0.27 \pm \\
0.03\end{array}$ & $\begin{array}{c}0.53 \pm \\
0.05\end{array}$ & $\begin{array}{c}49.34 \pm \\
0.48\end{array}$ & $\begin{array}{c}2.49 \pm \\
0.20\end{array}$ & $\begin{array}{c}19.89 \pm \\
1.70\end{array}$ & 15.59 & $\begin{array}{c}24.53 \pm \\
2.77\end{array}$ & $\begin{array}{c}217.14 \pm \\
9.90\end{array}$ & $\begin{array}{c}9.49 \pm \\
1.47\end{array}$ \\
\hline Croto nsuaveolens & $\begin{array}{c}75.62 \pm \\
3.67\end{array}$ & $\begin{array}{c}0.22 \pm \\
0.09\end{array}$ & $\begin{array}{c}2.43 \pm \\
0.14\end{array}$ & $\begin{array}{c}45.17 \pm \\
0.35\end{array}$ & $\begin{array}{c}2.33 \pm \\
0.53\end{array}$ & $\begin{array}{c}20.16 \pm \\
4.52\end{array}$ & 14.58 & $\begin{array}{c}26.87 \pm \\
1.66\end{array}$ & $\begin{array}{c}229.13 \pm \\
24.25\end{array}$ & $\begin{array}{c}34.55 \pm \\
4.11\end{array}$ \\
\hline Eryobotria japonica & $\begin{array}{c}18.77 \pm \\
1.68\end{array}$ & $\begin{array}{c}1.78 \pm \\
0.36\end{array}$ & $\begin{array}{c}2.20 \pm \\
0.20\end{array}$ & $\begin{array}{c}47.98 \pm \\
1.18\end{array}$ & $\begin{array}{c}3.03 \pm \\
0.35\end{array}$ & $\begin{array}{c}15.98 \pm \\
1.58\end{array}$ & 18.94 & $\begin{array}{c}22.04 \pm \\
3.44\end{array}$ & $\begin{array}{c}177.91 \pm \\
13.45\end{array}$ & $\begin{array}{c}17.13 \pm \\
1.56\end{array}$ \\
\hline Hedeoma palmeri & $\begin{array}{c}76.50 \pm \\
1.42\end{array}$ & $\begin{array}{c}0.18 \pm \\
0.14\end{array}$ & $\begin{array}{c}1.40 \pm \\
0.03\end{array}$ & $\begin{array}{c}46.38 \pm \\
1.66\end{array}$ & $\begin{array}{c}2.83 \pm \\
0.78\end{array}$ & $\begin{array}{c}17.14 \pm \\
3.33\end{array}$ & 17.69 & $\begin{array}{c}23.98 \pm \\
1.20\end{array}$ & $\begin{array}{c}334.23 \pm \\
9.96\end{array}$ & $\begin{array}{c}53.54 \pm \\
2.88\end{array}$ \\
\hline Marrubium vulgare & $\begin{array}{c}91.27 \pm \\
3.70\end{array}$ & $\begin{array}{c}0.64 \pm \\
0.42\end{array}$ & $\begin{array}{c}1.85 \pm \\
0.05\end{array}$ & $\begin{array}{c}40.48 \pm \\
0.32\end{array}$ & $\begin{array}{c}4.56 \pm \\
0.58\end{array}$ & $\begin{array}{c}8.99 \pm \\
1.03\end{array}$ & 28.48 & $\begin{array}{c}25.14 \pm \\
1.07\end{array}$ & $\begin{array}{c}374.78 \pm \\
13.18\end{array}$ & $\begin{array}{c}46.79 \pm \\
3.07\end{array}$ \\
\hline Melia azadirachta & $\begin{array}{c}90.99 \pm \\
7.21\end{array}$ & $\begin{array}{c}3.41 \pm \\
0.80\end{array}$ & $\begin{array}{c}1.98 \pm \\
0.35\end{array}$ & $\begin{array}{c}45.12 \pm \\
0.87\end{array}$ & $\begin{array}{c}5.85 \pm \\
0.32\end{array}$ & $\begin{array}{c}7.73 \pm \\
0.32\end{array}$ & 36.55 & $\begin{array}{c}24.20 \pm \\
5.26\end{array}$ & $\begin{array}{c}265.59 \pm \\
21.75\end{array}$ & $\begin{array}{c}52.57 \pm \\
11.28\end{array}$ \\
\hline Moringa oleifer & $\begin{array}{c}95.59 \pm \\
7.56\end{array}$ & $\begin{array}{c}0.81 \pm \\
0.89\end{array}$ & $\begin{array}{c}1.91 \pm \\
0.15\end{array}$ & $\begin{array}{c}45.96 \pm \\
0.23\end{array}$ & $\begin{array}{c}6.25 \pm \\
0.25\end{array}$ & $\begin{array}{c}7.37 \pm \\
0.31\end{array}$ & 39.05 & $\begin{array}{c}10.59 \pm \\
2.22\end{array}$ & $\begin{array}{c}773.04 \pm \\
198.33\end{array}$ & $\begin{array}{c}26.74 \pm \\
4.75\end{array}$ \\
\hline Opuntia ficus-indica & $\begin{array}{c}101.47 \pm \\
9.19\end{array}$ & $\begin{array}{c}6.39 \pm \\
0.90\end{array}$ & $\begin{array}{c}0.84 \pm \\
0.07\end{array}$ & $\begin{array}{c}25.54 \pm \\
0.99\end{array}$ & $\begin{array}{c}2.36 \pm \\
0.43\end{array}$ & $\begin{array}{l}11.1 \pm \\
1.94\end{array}$ & 14.74 & $\begin{array}{c}22.76 \pm \\
1.66\end{array}$ & $\begin{array}{c}135.18 \pm \\
10.44\end{array}$ & $\begin{array}{c}50.05 \pm \\
4.80\end{array}$ \\
\hline Phoradendron villosum & $\begin{array}{c}100.58 \pm \\
7.63\end{array}$ & $\begin{array}{c}2.29 \pm \\
0.68\end{array}$ & $\begin{array}{c}2.40 \pm \\
0.04\end{array}$ & $\begin{array}{c}40.4 \pm \\
0.63\end{array}$ & $\begin{array}{c}4.92 \pm \\
0.20\end{array}$ & $\begin{array}{c}8.22 \pm \\
0.44\end{array}$ & 30.76 & $\begin{array}{c}25.30 \pm \\
1.28\end{array}$ & $\begin{array}{c}151.30 \pm \\
10.06\end{array}$ & $\begin{array}{c}52.02 \pm \\
6.85\end{array}$ \\
\hline Rhus virens & $\begin{array}{c}14.77 \pm \\
2.64\end{array}$ & $\begin{array}{c}0.33 \pm \\
0.15\end{array}$ & $\begin{array}{c}1.41 \pm \\
0.26\end{array}$ & $\begin{array}{c}50.34 \pm \\
0.59\end{array}$ & $\begin{array}{c}2.27 \pm \\
0.45\end{array}$ & $\begin{array}{c}22.92 \pm \\
4.67\end{array}$ & 14.19 & $\begin{array}{c}22.98 \pm \\
6.24\end{array}$ & $\begin{array}{c}98.28 \pm \\
23.19\end{array}$ & $\begin{array}{c}12.75 \pm \\
1.54\end{array}$ \\
\hline Salix lasiolepis & $\begin{array}{c}15.66 \pm \\
3.34\end{array}$ & $\begin{array}{c}1.24 \pm \\
0.17\end{array}$ & $\begin{array}{c}1.19 \pm \\
0.07\end{array}$ & $\begin{array}{c}33.37 \pm \\
4.58\end{array}$ & $\begin{array}{c}2.06 \pm \\
0.50\end{array}$ & $\begin{array}{c}16.24 \pm \\
9.16\end{array}$ & 12.88 & $\begin{array}{c}8.73 \pm \\
3.27\end{array}$ & $\begin{array}{c}444.82 \pm \\
24.76\end{array}$ & $\begin{array}{c}216.31 \pm \\
10.83\end{array}$ \\
\hline Tecoma stans & $\begin{array}{c}57.22 \pm \\
8.90\end{array}$ & $\begin{array}{c}0.31 \pm \\
0.11\end{array}$ & $\begin{array}{c}1.36 \pm \\
0.02\end{array}$ & $\begin{array}{c}48.79 \pm \\
1.21\end{array}$ & $\begin{array}{c}3.28 \pm \\
0.47\end{array}$ & $\begin{array}{c}15.17 \pm \\
2.34\end{array}$ & 20.47 & $\begin{array}{c}25.67 \pm \\
3.33\end{array}$ & $\begin{array}{c}263.66 \pm \\
32.88\end{array}$ & $\begin{array}{c}29.49 \pm \\
1.27\end{array}$ \\
\hline Acacia wrightii & $\begin{array}{c}12.23 \pm \\
2.97\end{array}$ & $\begin{array}{c}0.49 \pm \\
0.28\end{array}$ & $\begin{array}{c}0.99 \pm \\
0.21\end{array}$ & $\begin{array}{c}36.59 \pm \\
1.11\end{array}$ & $\begin{array}{c}3.96 \pm \\
0.18\end{array}$ & $\begin{array}{c}9.25 \pm \\
6.22\end{array}$ & 24.75 & $\begin{array}{c}11.51 \pm \\
12.73\end{array}$ & $\begin{array}{c}196.38 \pm \\
15.07\end{array}$ & $\begin{array}{c}11.53 \pm \\
6.33\end{array}$ \\
\hline Acalypha monostachya & $\begin{array}{c}25.70 \pm \\
1.86\end{array}$ & $\begin{array}{c}8.11 \pm \\
0.88\end{array}$ & $\begin{array}{c}1.58 \pm \\
0.04\end{array}$ & $\begin{array}{c}29.71 \pm \\
3.28\end{array}$ & $\begin{array}{c}1.86 \pm \\
0.26\end{array}$ & $\begin{array}{c}15.95 \pm \\
12.53\end{array}$ & 11.64 & $\begin{array}{c}9.80 \pm \\
1.50\end{array}$ & $\begin{array}{c}275.96 \pm \\
36.79\end{array}$ & $\begin{array}{c}64.74 \pm \\
3.01\end{array}$ \\
\hline Chrysactinia Mexicana & $\begin{array}{l}5.47 \pm \\
0.81\end{array}$ & $\begin{array}{l}0.57 \pm \\
0.36\end{array}$ & $\begin{array}{l}0.86 \pm \\
0.05\end{array}$ & $\begin{array}{l}45.04 \pm \\
0.48\end{array}$ & $\begin{array}{l}3.39 \pm \\
0.49\end{array}$ & $\begin{array}{l}13.56 \pm \\
2.46\end{array}$ & 21.2 & $\begin{array}{l}12.53 \pm \\
1.69\end{array}$ & $\begin{array}{l}231.53 \pm \\
21.08\end{array}$ & $\begin{array}{l}30.30 \pm \\
3.09\end{array}$ \\
\hline Cnidoscolus aconitifolius & $\begin{array}{l}39.96 \pm \\
0.98\end{array}$ & $\begin{array}{l}2.28 \pm \\
0.36\end{array}$ & $\begin{array}{l}2.05 \pm \\
0.06\end{array}$ & $\begin{array}{l}39.11 \pm \\
2.20\end{array}$ & $\begin{array}{l}4.34 \pm \\
0.08\end{array}$ & $\begin{array}{l}9.02 \pm \\
2.75\end{array}$ & 27.09 & $\begin{array}{l}29.67 \pm \\
1.04\end{array}$ & $\begin{array}{l}354.72 \pm \\
20.59\end{array}$ & $\begin{array}{l}52.01 \pm \\
4.65\end{array}$ \\
\hline
\end{tabular}




\begin{tabular}{|c|c|c|c|c|c|c|c|c|c|c|}
\hline \multirow[t]{3}{*}{ Plant Species } & \multicolumn{5}{|c|}{ Leaf Macro-nutrient Content } & \multirow[t]{3}{*}{$\mathrm{C}: \mathrm{N}$} & \multirow{3}{*}{$\begin{array}{c}\% \\
\text { Pro- } \\
\text { teina }\end{array}$} & \multirow{2}{*}{\multicolumn{3}{|c|}{$\frac{\text { Leaf Micro-nutrient Content }}{\left(\mu \mathrm{g} \mathrm{g}^{-1} \mathrm{dw}\right)}$}} \\
\hline & \multicolumn{3}{|c|}{$(\mathrm{mg} g-1 \mathrm{dw})$} & \multicolumn{2}{|c|}{$(\%)$} & & & & & \\
\hline & $\mathrm{K}$ & $\mathrm{Mg}$ & $\mathrm{P}$ & $\mathrm{C}$ & $\mathrm{N}$ & & & $\mathrm{Cu}$ & $\mathrm{Fe}$ & $\mathrm{Zn}$ \\
\hline Dyssodia setifolia & $\begin{array}{l}11.12 \pm \\
2.24\end{array}$ & $\begin{array}{l}0.58 \pm \\
0.44\end{array}$ & $\begin{array}{l}1.06 \pm \\
0.29\end{array}$ & $\begin{array}{l}39.68 \pm \\
1.35\end{array}$ & $\begin{array}{l}2.35 \pm \\
0.67\end{array}$ & $\begin{array}{l}18.59 \pm \\
7.38\end{array}$ & 14.67 & $\begin{array}{l}25.25 \pm \\
1.45\end{array}$ & $\begin{array}{l}3540.19 \pm \\
557.14 ? ?\end{array}$ & $\begin{array}{l}27.97 \pm \\
1.25\end{array}$ \\
\hline Equisetum hyemale & $\begin{array}{l}12.86 \pm \\
0.86\end{array}$ & $\begin{array}{l}2.28 \pm \\
0.38\end{array}$ & $\begin{array}{l}1.51 \pm \\
0.61\end{array}$ & $\begin{array}{l}26.95 \pm \\
1.09\end{array}$ & $\begin{array}{l}1.81 \pm \\
0.08\end{array}$ & $\begin{array}{l}14.89 \pm \\
12.82\end{array}$ & 11.31 & $\begin{array}{l}9.97 \pm \\
1.63\end{array}$ & $\begin{array}{l}229.29 \pm \\
8.67\end{array}$ & $\begin{array}{l}107.44 \pm \\
17.64\end{array}$ \\
\hline Eruca sativa & $\begin{array}{l}144.23 \pm \\
2.30\end{array}$ & $\begin{array}{l}2.22 \pm \\
0.24\end{array}$ & $\begin{array}{l}2.22 \pm \\
0.26\end{array}$ & $\begin{array}{l}41.13 \pm \\
0.72\end{array}$ & $\begin{array}{l}5.48 \pm \\
0.64\end{array}$ & $\begin{array}{l}7.59 \pm \\
0.90\end{array}$ & 34.23 & $\begin{array}{l}22.54 \pm \\
8.27\end{array}$ & $\begin{array}{l}312.46 \pm \\
60.02\end{array}$ & $\begin{array}{l}45.51 \pm \\
1.96\end{array}$ \\
\hline Eryngium heterophyllum & $\begin{array}{l}43.07 \pm \\
8.87\end{array}$ & $\begin{array}{l}0.38 \pm \\
0.29\end{array}$ & $\begin{array}{l}2.27 \pm \\
0.11\end{array}$ & $\begin{array}{l}40.9 \pm \\
0.65\end{array}$ & $\begin{array}{l}1.75 \pm \\
0.40\end{array}$ & $\begin{array}{l}24.23 \pm \\
4.89\end{array}$ & 10.94 & $\begin{array}{l}33.30 \pm \\
12.18\end{array}$ & $\begin{array}{l}144.26 \pm \\
57.95\end{array}$ & $\begin{array}{l}15.45 \pm \\
3.49\end{array}$ \\
\hline Eugenia caryophyllata & $\begin{array}{l}37.73 \pm \\
1.40\end{array}$ & $\begin{array}{l}0.97 \pm \\
0.34\end{array}$ & $\begin{array}{l}0.89 \pm \\
0.08\end{array}$ & $\begin{array}{l}51.66 \pm \\
1.85\end{array}$ & $\begin{array}{l}2.9 \pm \\
0.35\end{array}$ & $\begin{array}{l}18.01 \pm \\
2.30\end{array}$ & 18.14 & $\begin{array}{l}27.33 \pm \\
1.25\end{array}$ & $\begin{array}{l}221.34 \pm \\
156.74\end{array}$ & $\begin{array}{l}12.27 \pm \\
0.56\end{array}$ \\
\hline Gnahalium canescens & $\begin{array}{l}16.69 \pm \\
0.53\end{array}$ & $\begin{array}{l}1.40 \pm \\
0.18\end{array}$ & $\begin{array}{l}1.08 \pm \\
0.26\end{array}$ & $\begin{array}{l}37.73 \pm \\
1.26\end{array}$ & $\begin{array}{l}2.56 \pm \\
0.32\end{array}$ & $\begin{array}{l}14.89 \pm \\
1.37\end{array}$ & 15.99 & $\begin{array}{l}25.13 \pm \\
1.70\end{array}$ & $\begin{array}{l}3973.55 \pm \\
1342.28\end{array}$ & $\begin{array}{l}53.73 \pm \\
0.79\end{array}$ \\
\hline Gochnatia hypoleuca & $\begin{array}{l}22.54 \pm \\
2.48\end{array}$ & $\begin{array}{l}0.48 \pm \\
0.25\end{array}$ & $\begin{array}{l}0.82 \pm \\
0.04\end{array}$ & $\begin{array}{l}49.86 \pm \\
0.87\end{array}$ & $\begin{array}{l}3.59 \pm \\
0.50\end{array}$ & $\begin{array}{l}14.11 \pm \\
1.89\end{array}$ & 22.41 & $\begin{array}{l}11.37 \pm \\
0.89\end{array}$ & $\begin{array}{l}292.92 \pm \\
23.68\end{array}$ & $\begin{array}{l}24.43 \pm \\
0.77\end{array}$ \\
\hline Lepidium virginicumm & $\begin{array}{l}11.67 \pm \\
0.59\end{array}$ & $\begin{array}{l}1.89 \pm \\
0.52\end{array}$ & $\begin{array}{l}2.27 \pm \\
0.05\end{array}$ & $\begin{array}{l}43.8 \pm \\
1.22\end{array}$ & $\begin{array}{l}4.46 \pm \\
0.59\end{array}$ & $\begin{array}{l}9.95 \pm \\
1.11\end{array}$ & 27.85 & $\begin{array}{l}5.36 \pm \\
1.80\end{array}$ & $\begin{array}{l}188.69 \pm \\
7.64\end{array}$ & $\begin{array}{l}28.26 \pm \\
1.49\end{array}$ \\
\hline Litsea glauscesens & $\begin{array}{l}10.94 \pm \\
2.47\end{array}$ & $\begin{array}{l}0.33 \pm \\
0.28\end{array}$ & $\begin{array}{l}2.40 \pm \\
0.13\end{array}$ & $\begin{array}{l}51.34 \pm \\
0.28\end{array}$ & $\begin{array}{l}3.36 \pm \\
0.45\end{array}$ & $\begin{array}{l}15.5 \pm \\
2.03\end{array}$ & 21 & $\begin{array}{l}22.84 \pm \\
7.79\end{array}$ & $\begin{array}{l}177.31 \pm \\
27.21\end{array}$ & $\begin{array}{l}33.09 \pm \\
2.68\end{array}$ \\
\hline Lepidium virginicumm & $\begin{array}{l}58.22 \pm \\
4.35\end{array}$ & $\begin{array}{l}1.15 \pm \\
0.24\end{array}$ & $\begin{array}{l}2.20 \pm \\
0.03\end{array}$ & $\begin{array}{l}44.14 \pm \\
2.71\end{array}$ & $\begin{array}{l}5.4 \pm \\
0.15\end{array}$ & $\begin{array}{l}8.18 \pm \\
0.41\end{array}$ & 33.73 & $\begin{array}{l}31.06 \pm \\
0.74\end{array}$ & $\begin{array}{l}373.38 \pm \\
9.40\end{array}$ & $\begin{array}{l}49.17 \pm \\
5.46\end{array}$ \\
\hline Mimosa malacophylla & $\begin{array}{l}16.39 \pm \\
0.45\end{array}$ & $\begin{array}{l}8.64 \pm \\
0.99\end{array}$ & $\begin{array}{l}0.84 \pm \\
0.04\end{array}$ & $\begin{array}{l}45.15 \pm \\
0.53\end{array}$ & $\begin{array}{l}8.46 \pm \\
0.18\end{array}$ & $\begin{array}{l}5.34 \pm \\
0.17\end{array}$ & 52.87 & $\begin{array}{l}4.17 \pm \\
1.25\end{array}$ & $\begin{array}{l}222.28 \pm \\
3.85\end{array}$ & $\begin{array}{l}35.28 \pm \\
1.14\end{array}$ \\
\hline Nicotiana glauca & $\begin{array}{l}18.19 \pm \\
1.32\end{array}$ & $\begin{array}{l}1.31 \pm \\
0.15\end{array}$ & $\begin{array}{l}1.30 \pm \\
0.12\end{array}$ & $\begin{array}{l}37.94 \pm \\
0.56\end{array}$ & $\begin{array}{l}4.79 \pm \\
0.54\end{array}$ & $\begin{array}{l}8.00 \pm \\
0.87\end{array}$ & 29.91 & $\begin{array}{l}33.06 \pm \\
2.95\end{array}$ & $\begin{array}{l}721.51 \pm \\
234.69\end{array}$ & $\begin{array}{l}43.64 \pm \\
3.96\end{array}$ \\
\hline Ocimum basilicum & $\begin{array}{l}72.09 \pm \\
2.43\end{array}$ & $\begin{array}{l}2.42 \pm \\
0.09\end{array}$ & $\begin{array}{l}2.18 \pm \\
0.03\end{array}$ & $\begin{array}{l}38.31 \pm \\
0.34\end{array}$ & $\begin{array}{l}4.66 \pm \\
0.45\end{array}$ & $\begin{array}{l}8.29 \pm \\
0.84\end{array}$ & 29.1 & $\begin{array}{l}14.56 \pm \\
0.79\end{array}$ & $\begin{array}{l}405.13 \pm \\
34.32\end{array}$ & $\begin{array}{l}118.77 \pm \\
5.93\end{array}$ \\
\hline Olea europea & $\begin{array}{l}48.16 \pm \\
1.21\end{array}$ & $\begin{array}{l}0.80 \pm \\
0.20\end{array}$ & $\begin{array}{l}1.23 \pm \\
0.11\end{array}$ & $\begin{array}{l}41.13 \pm \\
2.59\end{array}$ & $\begin{array}{l}1.86 \pm \\
0.8\end{array}$ & $\begin{array}{l}22.08 \pm \\
3.23\end{array}$ & 11.64 & $\begin{array}{l}7.91 \pm \\
1.13\end{array}$ & $\begin{array}{l}406.30 \pm \\
31.86\end{array}$ & $\begin{array}{l}21.93 \pm \\
0.82\end{array}$ \\
\hline Phalaris canariensis & $\begin{array}{l}163.35 \pm \\
6.88\end{array}$ & $\begin{array}{l}2.74 \pm \\
0.30\end{array}$ & $\begin{array}{l}1.48 \pm \\
0.17\end{array}$ & $\begin{array}{l}40.73 \pm \\
0.53\end{array}$ & $\begin{array}{l}2.84 \pm \\
0.52\end{array}$ & $\begin{array}{l}14.78 \pm \\
2.87\end{array}$ & 17.73 & $\begin{array}{l}33.78 \pm \\
8.95\end{array}$ & $\begin{array}{l}254.84 \pm \\
70.34\end{array}$ & $\begin{array}{l}63.97 \pm \\
7.56\end{array}$ \\
\hline Poliomintha longiflora & $\begin{array}{l}31.63 \pm \\
5.50\end{array}$ & $\begin{array}{l}1.80 \pm \\
0.81\end{array}$ & $\begin{array}{l}1.45 \pm \\
0.75\end{array}$ & $\begin{array}{l}42.9 \pm \\
0.24\end{array}$ & $\begin{array}{l}4.89 \pm \\
0.21\end{array}$ & $\begin{array}{l}8.79 \pm \\
0.36\end{array}$ & 30.54 & $\begin{array}{l}29.04 \pm \\
2.79\end{array}$ & $\begin{array}{l}316.63 \pm \\
130.27\end{array}$ & $\begin{array}{l}32.99 \pm \\
7.99\end{array}$ \\
\hline Rosamrinus officinalis & $\begin{array}{l}26.59 \pm \\
5.54\end{array}$ & $\begin{array}{l}1.18 \pm \\
0.28\end{array}$ & $\begin{array}{l}1.24 \pm \\
0.38\end{array}$ & $\begin{array}{l}47.77 \pm \\
5.43\end{array}$ & $\begin{array}{l}4.54 \pm \\
0.24\end{array}$ & $\begin{array}{l}10.57 \pm \\
1.65\end{array}$ & 28.41 & $\begin{array}{l}11.36 \pm \\
7.39\end{array}$ & $\begin{array}{l}336.95 \pm \\
122.63\end{array}$ & $\begin{array}{l}86.07 \pm \\
21.27\end{array}$ \\
\hline Ruta graveolens & $\begin{array}{l}28.04 \pm \\
2.59\end{array}$ & $\begin{array}{l}0.88 \pm \\
0.20\end{array}$ & $\begin{array}{l}1.24 \pm \\
0.20\end{array}$ & $\begin{array}{l}38.86 \pm \\
1.41\end{array}$ & $\begin{array}{l}2.79 \pm \\
0.25\end{array}$ & $\begin{array}{l}13.23 \pm \\
5.72\end{array}$ & 17.41 & $\begin{array}{l}20.87 \pm \\
9.55\end{array}$ & $\begin{array}{l}458.62 \pm \\
71.30\end{array}$ & $\begin{array}{l}50.72 \pm \\
11.64\end{array}$ \\
\hline Salvia hispanica & $\begin{array}{l}46.48 \pm \\
6.70\end{array}$ & $\begin{array}{l}3.52 \pm \\
0.71\end{array}$ & $\begin{array}{l}5.79 \pm \\
0.75\end{array}$ & $\begin{array}{l}44.68 \pm \\
2.18\end{array}$ & $\begin{array}{l}5.24 \pm \\
0.62\end{array}$ & $\begin{array}{l}8.59 \pm \\
0.81\end{array}$ & 32.77 & $\begin{array}{l}28.11 \pm \\
4.75\end{array}$ & $\begin{array}{l}479.51 \pm \\
64.24\end{array}$ & $\begin{array}{l}62.22 \pm \\
3.93\end{array}$ \\
\hline Tagetes lucida & $\begin{array}{l}21.49 \pm \\
3.31\end{array}$ & $\begin{array}{l}1.90 \pm \\
0.13\end{array}$ & $\begin{array}{l}1.40 \pm \\
0.04\end{array}$ & $\begin{array}{l}46.19 \pm \\
1.04\end{array}$ & $\begin{array}{l}5.89 \pm \\
0.29\end{array}$ & $\begin{array}{l}7.85 \pm \\
0.33\end{array}$ & 36.81 & $\begin{array}{l}8.93 \pm \\
2.40\end{array}$ & $\begin{array}{l}167.40 \pm \\
13.81\end{array}$ & $\begin{array}{l}12.16 \pm \\
1.64\end{array}$ \\
\hline Tillandsiausenoides L. & $\begin{array}{l}40.56 \pm \\
4.67\end{array}$ & $\begin{array}{l}1.20 \pm \\
0.14\end{array}$ & $\begin{array}{l}4.45 \pm \\
0.17\end{array}$ & $\begin{array}{l}44.10 \pm \\
1.61\end{array}$ & $\begin{array}{l}1.56 \pm \\
0.71\end{array}$ & $\begin{array}{l}31.32 \pm \\
8.20\end{array}$ & 9.75 & $\begin{array}{l}31.70 \pm \\
24.04\end{array}$ & $\begin{array}{l}936.75 \pm \\
543.65\end{array}$ & $\begin{array}{l}34.06 \pm \\
9.45\end{array}$ \\
\hline Tragia ramosa & $\begin{array}{l}60.57 \pm \\
10.12\end{array}$ & $\begin{array}{l}0.46 \pm \\
0.42\end{array}$ & $\begin{array}{l}1.45 \pm \\
0.10\end{array}$ & $\begin{array}{l}42.68 \pm \\
1.16\end{array}$ & $\begin{array}{l}3.89 \pm \\
0.63\end{array}$ & $\begin{array}{l}11.22 \pm \\
2.01\end{array}$ & 24.31 & $\begin{array}{l}24.96 \pm \\
2.87\end{array}$ & $\begin{array}{l}1450.25 \pm \\
273.89\end{array}$ & $\begin{array}{l}57.87 \pm \\
5.81\end{array}$ \\
\hline
\end{tabular}


absorption of plants (Linderoth et al., 2002).

An indigenous Malaysian herb, Karcip Fatimah (Lobisia pumila Blume) used in South East Asia is found to possess health promoting properties The high production of secondary metabolites and antioxidants were highly correlated to low nitrogen content and high $\mathrm{C} / \mathrm{N}$ ratio showing correlation between secondary metabolites and antioxidant activity. Therefore the consumption of L. pumila promotes several antioxidant activities (Ibrahim and Jaafar, 2011). Some plants with high $\mathrm{C} / \mathrm{N}$ produce secondary metabolites and antioxidants necessary for old age and high medicinal value (Linderoth et al., 2002) High $\mathrm{C} / \mathrm{N}$ has been reported in Lobsia pumila (Ibrahim and Jaafar, 2011) and in Aconitum naviculare (Brühl) Stapf and Neopicrorhizascrophulariiflor.

Protein is useful to improve enzymatic activity and improve health. Mimosa malacophylla (52.87), Moringa oleIfer (39.04), Tagetes lucida (36.81), Poliomintha longiflora (36.54), Eruca sativa (34.23), Lepidium virginicumm (33.73), Melia azadirachta (36.55), Nicotiana glauca (29.1), Phoradendron villosum (30.76) are the important species exhibit higher protein.

\subsection{Micronutrients}

Copper is needed for stimulating antioxidant reactions, as well as to produce enzymes for a number of body functions. Some of the species containing good amount of Cupper are Celtis laevigata (33.88), Phalaris canariensis (33.78), Tillandsia usenoides (33.70), Arbutus xalapensis (33.40), Lepidium virginicumm (33.06), Phalaris canariensis (33.04), Lepidium virginicumm (31.06), Lepidium virginicumm (31.06), Buddleja cordata (29.90), Cnidoscolus aconitifolius (29.67).

Iron requirement is very essential as it not only forms a part of protein haemoglobin an oxygen carrying pigment also forms a part of many enzymes involved in an array of cell functions. Gnahalium canescens (39777.55), Tragia ramosa (1450.25), Tillandsia usenoides (936.75), Nicotiana glauca (771.51), Salvia hispanica (479.51), Salix lasiolepis (444.872) Ruta graveolens (458.62), Olea europea (406.30), Ocimum basilicum (405.13), Marrubium vulgare (374.78), Lepidium virginicumm (373.38), Cnidoscolus aconitifolius (354.47), Arbutus xalapensis (347.58), Rosamrinus officinalis (336.95), Hedeoma palmeri (334.23), Poliomintha longiflora (316.63), Eruca sativa (312.46) are the plants with higher Iron.

Zinc is required for normal growth, a healthy immune system function, for new protein synthesis, DNA synthesis and cell division as well for different chemical reactions. The species with higher Zinc are Salix lasiolepis (216.31), Ocimum basilicum (118.77), Rosamrinus officinalis (86.07), Salvia hispanica (62.22), Phalaris canariensis (63.97), Tragia ramosa (57.87), Carya illinoiensis (57.69), Agave macroculmis
(55.20), Gnahalium canescens (53.73), Hedeoma palmeri (53.54), Melia azadirachta (52.57), Ruta graveolens (50.72), Opuntiaficus-indica (50.05), Phoradendron villosum (52.02).

Overall, Meringa oleir contain K (95.59 $\left.\mathrm{mg} \mathrm{g}^{-1} \mathrm{dw}\right), \mathrm{C}(45.90 \%$ $\left.\mathrm{mg} \mathrm{g}^{-1} \mathrm{dw}\right), \mathrm{N}\left(6.25 \mathrm{mg} \mathrm{g}^{-1} \mathrm{dw}\right)$, protein $(39.05 \%), \mathrm{Fe}(777.04$ $\left.\mathrm{mg} \mathrm{g}^{-1} \mathrm{dw}\right), \mathrm{Zn}\left(26.7 \mathrm{mg} \mathrm{g}^{-1} \mathrm{dw}\right)$ Opuntia ficus-indica contain $\mathrm{K}$ (104.47 $\left.\mathrm{mg} \mathrm{g}^{-1} \mathrm{dw}\right), \mathrm{Mg}\left(6.39 \mathrm{mg} \mathrm{g}^{-1} \mathrm{dw}\right)$, Fe (135.18 $\mathrm{mg} \mathrm{g}^{-1}$ $\mathrm{dw}), \mathrm{Zn}\left(30.01 \mathrm{mg} \mathrm{g}^{-1} \mathrm{dw}\right)$ Melia azadirachta contain K (90.99 $\left.\mathrm{mg} \mathrm{g}^{-1} \mathrm{dw}\right), \mathrm{Mg}\left(3.41 \mathrm{mg} \mathrm{g}^{-1} \mathrm{dw}\right), \mathrm{C}\left(45.13 \mathrm{mg} \mathrm{g}^{-1} \mathrm{dw}\right), \mathrm{N}$ (3.85) $\mathrm{mg} \mathrm{g}^{-1} \mathrm{dw}$, Protein (39.03), Fe (773.04) $\mathrm{mg} \mathrm{g}^{-1} \mathrm{dw}$. Tecoma stans contain $\mathrm{K}$ (57.22 $\left.\mathrm{mg} \mathrm{g}^{-1} \mathrm{dw}\right), \mathrm{C}(48.79 \%)$, protein $(20.47 \%)$, $\mathrm{Cu}$ (23.66) $\mathrm{mg} \mathrm{g}^{-1} \mathrm{dw}$, Fe (263.63 $\left.\mathrm{mg} \mathrm{g}^{-1} \mathrm{dw}\right)$.

Several medicinal plants possess micronutrients are useful in diabetes (Maiti et al., 2015). Few medicinal plants are reported to be effective to control blood sugar such as Mulberry leaf, soybean, black bean, Persian shallot, Zizyphus lotus, etc. Some Mexican plants contain $\beta$-glycosides and other compounds that are effective in lowering blood sugar and body weight in diabetic patients.

The medicinal plants used traditionally by Mexicans as include 19 species for diabetes, few for stomach ache, and others in various diseases such as cancer, bronchitis, infertility, inflammation, blood circulation, digestion, fever, kidney problem, earache, cholesterol, cough, respiratory headache, circulation, burning (Estrada et al., 2007). The supply of macroand micronutrients has been found to useful in alleviating various diseases. Several medicinal plants used to reduce blood sugar such as Mulberry leaf, soybean, black bean, Persian shallot, Zizyphus lotus, etc, such as in alleviating eye problem (Kowluru et al., 2008); diabetes (Patton et al., 2007, Farvid et al., 2011).

In the present study, we determined macro-and micronutrients and protein contents of 44 medicinal plants used to treat various diseases, with an objective to select species of high nutrient profile and nutraceuticalva uses irrespective of their uses in various diseases.

Different micronutrients, although required in minor quantities are essential for good health of mankind and animals. The deficiency of these elements causes abnormalities leading to infection of diseases. In addition to micronutrients some major elements are necessary in higher amounts for good health. In this respect phosphorus is required for many biochemical reactions It in the body, such as conversion of foods to energy, muscle contractions, nerve conductions, normal kidney functions and helps to build strong bones. It is observed that most of the species used traditionally to combat various diseases contain very high amount of macro and micronutrients and protein confirming the efficacy of these species. In this respect we have no scope to compare the contents of nutrient 
profile with individual disease species wise. In the case of diabetes we want to mention the medicinal efficacy of few species of few species commonly used in combating diabetes.

Although various medicinal plant species are used traditionally to combat various disease but the efficacy of individual species is not scientifically confirmed through for level of tolerance in laboratory tests. On the other hand, although few studies are available on phyto chemistry of medicinal plants to confirm their efficacy, no systematic studies have been undertaken on the nutritional values (macro-and micronutrients, protein content) of the medicinal plants.

In this context research on nutraceutical plants is a modern trend. The nutraceutical values of various plants is well documents in the literature (It is evident from this study that the 4 species which are commonly used to combat diabetes contain very high amount of nutrients, there by confirming their efficacy, but the efficacy of these species should be confirmed by analysing the tolerance level in a pharmacology laboratory. On the other hand, may be cited the use of Tragia ramose commonly used traditionally in in blood purification contain very high amount of $\mathrm{Fe}$ (1250.25) confirming the role of iron as blood purifier and haemoglobin function. This need be confirmed pharmacologically. On the other hand, the exceptional high amount of iron in the case of Dyssodia setifolia, containing 3540.19 could be confirmed for its possible efficacy in blood purification, haemoglobin function and blood circulation through pharmacological study.

Most of the medicinal plant species used in the present study contains enriched sources of nutraceutical which need to be harnessed. Very recently strong recommendations for consumption of nutraceutical from plant have become popular to improve health and to prevent and treat various diseases. Some popular phyto-nutraceuticals include glucosamine from ginseng Omega 3 fatty acids from linseed. Many of nutraceutical have high potentials for multiple the rapetic, but research is lacking (Pandey et al., 2011).

\section{Conclusion}

Based on the analysis of macro and micro nutrients the following species Phalaris canariensis, Eruc asativa, Ocimum basilicum, Tragia ramosa, Mentha piperita, Mimosa malacophylla, Acalypha monostachya, Salvia hispanica, Tillandsia usenoides, Letsea glauscens, Tagetes lucida, Erygium heterophylla, Dyssodia setifolia, Nicotiana glauca,,Ruta graveolens, Olea europea, Equisetum hyemale, Rosamrinus officinalisare selected containing a majority of each of these nutrients. In our study, we obtained few species with high $\mathrm{C} / \mathrm{N}$ ratio having high potentialities of the production of secondary metabolites and antioxidants exhibiting as the potential lines of research in future.

\section{Acknowledgement}

The authors are highly thankful to Elsa Gonzalez for dedicated hard work in the chemical analysis of nutrients of the medicinal plants to our full satisfaction.

\section{References}

Agrawal, S.B., R, Kumari., 2013. Assessment of litter degradation in medicinal plants subjected to ultraviolet-B radiation. Journal of Environmental Biology 34(4), 739-745.

Bell, W.H., Castetter, E.F., 1937. The utilization of mesquite and screw bean by the aborigines in the American southwest University New Mexico Bulletin 314, Biological Series 5(2), 1-55.

Cherney, D.J.R., 2000. Characterization of forages by chemical analysis. Ch 14. In: Givens, D.I., Owen, E., Axford, R.F.E., Ohmed, H.M. (Eds.), Forage evaluation in ruminant nutrition, 281-300. CABI Publishing, Wallingford, UK.

Devangyam, T.P.A., Tilak, J.C., Boloor, K.K., Sane, K.S., Ghaskadbi, S.S. Lele, R.D., 2004. Free radical and antioxidants: Current status and future prospects. Journal of the Association of Physicians of India 52, 794-804.

Farvid, M.S., Homayouni, F., Amiri, Z., Adelmanesh, F., 2011. Improving neuropathy scores in type 2 diabetic patients using micronutrients supplementation. Diabetes research and Clinical Practice, 93(1), 86-94.

Halliwal, B., Gutteridge, J.M.C., 1989. Free radicles in Biology and Medicine. $2^{\text {nd }}$ Edn. Clarendon Press, Oxford, UK.

Hernandez-Sandoval, L., Gonzalez, C., Gonzalez-Medrano, F., 1991. Plantasutiles de Tamaulipas, Mexico. Analesdel Instituto de BilogIa de la Universidad Nacional Autonoma de Mexico Serie Botanica 62(1), 1-38.

Ibrahim, M.H., Jaafar, H.Z.E., 2011. The relationship of nitrogen and $\mathrm{C} / \mathrm{N}$ ratio with secondary metabolites levels and antioxidant activities in three varieties of Malaysian Karcip Fatimah (Lobisia pumulla Blume). Molecules $16,5514-5526$.

Ibrahim, M.H., Jaafar, H.Z.E., Rahmat, A., Rahman, Z.A., 2011. The relationship between phenolics and flavonoids production with total non-structural carbohydrate and photosynthetic rate in Lobisia plumula Benth under high $\mathrm{CO}_{2}$ and nitrogen fertilization, Molecules 16, 162-174.

Kowluru, R., A., Kanwar, M., Chan, P., Zhang, J.P., 2008. Inhibition of retinopathy and retinal metabolic abnormalities in diabetic rats with AREDS-based micronutrients. Archives of ophthalmology 126(9), 
$1266-1272$

Lindroth, R.L., Osier, T.L., Burnhill, H.R.H., Wood, S.A. 2002. Effects of genotype and nutrient availability on photosensitivity of Trembling aspen (Populus tremoides Mich) during leaf senescence. Biochemical Systematics and Ecology 30, 297-307.

Maiti, R.K., Rodriguez, H.R., Kumari, A., Sarkar, N.C., 2016. Macro and micronutrient contents of 18 medicinal plants used traditionally in Nuevo Leon, Northeast Mexico. Pakistan Journal of Botany 48(1), 271-276.

Mittermeier, R., Mittermeier, C.G.de., 1992. La importancia de la biodiversidad biologica de Mexico. In: Sarukhan, J., Dirzo, R. (Eds.), Mexico ante los retos de la biodiversidad. Comision Nacional para Conocimiento y Uso de la Biodiversidad. Mexico, 43-55.

Mittermeier, R.A., 1988. Primate diversity and the tropical forest. In: Wilson E.O., Peter, F.M. (Eds.), Biodiversity. National Academy Press, Washington DC., 145-154.

Mondel, S., Youdin, M.B., 2004.Catechin polyphenol: Neurodegration and neuroprotection in neurological diseases. Free Radical Biology and Medicine 37, 304-317.

Pandey, N., Meena, R., Rai, S.K., Rai, S, P., 2011. Medicinal plants derived nutraceutical: A remerging health aid. International Journal of Plants and Biosciences 2, 419-442.

Patton, S.R., Dolan, L.M., Powers,S.W., 2007. Dietary adherence and associated glycemic control in families of young children with type 1 Diabetes. Journal of the Academy of Nutrition and Diebetics 107(1), 46-52.

Sarfini, M., Bellecco, M. Wolk, A., Ekstrom, A.M., 2002, Total antioxidants potential of fruit and vegetables and risk of gastric cancer. Gatrometeorology 123, 985-991.

Schramm, D.D., Karim, M., Schrador, H.R., Holt, R.R., Cardeth, M., Keen, C.L., 2003. Honey with high levels of antioxidants can provide protection to healthy human subjects. Journal of Agricultural and Food Chemistry 51, 1792-1935.

Shaheen, S.O., Sterne, J.A., Thompson, R.I., Songhurst, C.E., Margetts, B.M., Burney, P.G., 2001. Dietary antioxidant and asthma in adults: Population-based case control study. American Journal of Respiratory and Critical Care Medicine 164, 1823-1828.

Shoskes, D.A., Zedinm, S.I., Shahed, A., Rajfi, J., 1999. Quercetin in men with category III chronic prostatitis: a preliminary prospective, double-blind, placebocontrolled trial. Urology, 54, 960-963.

Vega-Avila, E., Espejo-Serna, A., Alarcon-Aguilar, F., Velasco-Lezama, R., 2009. Cytotoxic activity of four Mexican medicinal plants. In: Proceedings of the West Pharmacology Society 52, 78-82.

Villasenor, J.L., 2003. Diversidad y distribucion de las Magnoliophyta de Mexico. Interciencia 28, 160-167. 